\title{
Ethyl Acetate Extract of Asclepias curassavica Induced Apoptosis in Human Cancer Cells via Activating p38 and JNK MAPK Signaling Pathways
}

\author{
Xi Zheng, ${ }^{1}$ Ying Xu, ${ }^{1}$ Bei Liu, ${ }^{2}$ Yan Qi, ${ }^{1}$ Ying Zhang, ${ }^{1}$ Xiaosi Li, ${ }^{1}$ Xia Zhang, ${ }^{2}$ Xiaojia Pu, \\ Siwen Li $\mathbb{D})^{1}$ Zhe Chen $\mathbb{D}^{1},{ }^{3}$ and Chunping Wan $\mathbb{D}^{1}$
}

${ }^{1}$ The No. 1 Affiliated Hospital of Yunnan University of Traditional Chinese Medicine, Kunming 650021, China

${ }^{2}$ School of Pharmacy, Yunnan University of Traditional Chinese Medicine, Kunming 650500, China

${ }^{3}$ Zhejiang Hospital of Traditional Chinese Medicine, Zhejiang Chinese Medical University, Hangzhou 310006, China

Correspondence should be addressed to Siwen Li; slststl@21cn.com, Zhe Chen; chenzhe@zju.edu.cn, and Chunping Wan; wanchunping1012@163.com

Received 11 September 2018; Revised 25 February 2019; Accepted 30 April 2019; Published 9 June 2019

Academic Editor: Youn-Chul Kim

Copyright (C) 2019 Xi Zheng et al. This is an open access article distributed under the Creative Commons Attribution License, which permits unrestricted use, distribution, and reproduction in any medium, provided the original work is properly cited.

\begin{abstract}
Background. Asclepias curassavica L. (Asclepiadaceae), as a traditional medicinal plant, is used as treatment for tumors in traditional Chinese and Indian medical practice. However, its underlying molecular mechanisms remain largely unresolved. The current study investigated its antitumor activity and the underlying molecular mechanisms. Method. Cell viability was detected by a real-time cell analysis system and MTT assay. Antitumor effect of ethyl acetate extract of Asclepias curassavica (EAAC) on NIC-H1975 tumors in vivo was assessed in BALB/c-nu/nu mouse. Apoptosis was measured using Hoechst33342 staining and Annexin V/PIstaining. Apoptosis-related proteins and MAPK signaling pathways were analyzed based on Western blot assay. Results. EAAC exhibited the highest cytotoxic activity in vitro than other polar parts. Meanwhile, EAAC could inhibit sensitive cell line NICH1975 proliferation in a concentration-dependent and time-dependent manner. Furthermore, EAAC had a significant inhibitory effect on NIC-H1975 tumor growth in BALB/c-nu/nu mouse. NIC-H1975 cells showed obvious apoptosis characteristics after EAAC treatment. Fas, caspase family members caspase 3, caspase 9, and caspase 8 showed dose-dependent induction by EAAC treatment, with increasing PARP cleavage. Additionally, EAAC significantly downregulated antiapoptotic proteins Bcl-2, XIAP, survivin, and Mcl-1 and upregulated proapoptosis proteins Bak, Bax, as well as activation of p38 and JNK MAPK signaling pathways. Moreover, inhibiting p38 and JNK MAPK by pharmacological inhibitors abrogated EAAC-induced apoptosis. Conclusion. Our data indicated that EAAC exerted potent antitumor effect both in vitro and in vivo by triggering the apoptotic pathway.
\end{abstract}

\section{Background}

Cancer has threaten human life and health worldwide; in particular lung, breast, and colon cancer are the three most common cancers. Conventional chemotherapy plays a key role in the treatment of these cancers; however the results of treatment are fairly unpleasant, which drives us to develop a safe and highly effective tumor-inhibiting agent.

Many plants have been reported to possess various bioactivities, including antitumor activity. Asclepias curassavica L. (Asclepiadaceae), a traditional medicinal plant from Dai medicine in the southwestern China, is widely used as treatment against tumor, hemostatic disorders, inflammation, and pain [1]. Among these, the antitumor activity already received intensive attention from pharmaceutical researchers. However, its molecular mechanism remains unclear.

Impaired apoptosis is one of the hallmarks of cancer, which contribute to tumor progression and resistance to conventional cancer therapy [2]. Induction of cell apoptosis is a common strategy for cancer therapy. Successful apoptosisinducing drugs lead tumor cells to die by directly turning on/off antiapoptotic pathway. Many evidences revealed turning on/off many death and survival genes is involved in this 
process. Fas and FasL have important roles in the external death receptor pathway; members of the Bcl-2 family, Bcl2 and Bax, play a significant role in the mitochondrialmediated cell death pathway [3-5]; in addition, cytochrome $\mathrm{C}$ releasing from mitochondria can activate caspase 9 , which in turn activated executioner caspase 3 via cleavage induction [6].

In the present study, we found that ethyl acetate extract of Asclepias curassavica L. (EAAC) exerted potent antitumor effect both in vitro and in vivo; EAAC also induced apoptosis in NIC-H1975 cell line, which was related to suppressing the expression of survival proteins, including Bcl-2, survivin, and upregulating the expression of proapoptosis proteins, (e.g., bak). In particular, the cleavage of caspase 3, caspase 9, caspase 8 , and Fas expression was significantly increased after EAAC treatment.

The mitogen-activated protein kinase (MAPK) superfamily consists of ERK, p38 kinase, and JNK which have been implicated in cell proliferation, differentiation, invasion, and apoptosis [7]. Generally, p38 and JNK contribute to the induction of apoptosis [8-10], whereas ERK pathway activation is important for cell survival. From current study, we confirmed the phosphorylation of p38 and JNK was remarkably induced by EAAC, which confirmed p38 and JNK MAPK signaling pathways were involved in EAAC inducedapoptosis.

\section{Methods}

2.1. Preparation of Extracts of Asclepias curassavica. The dried aerial parts of Asclepias curassavica (5kg) were boiled in water (30min/time, 4 times), the water broth was extracted with chloroform, ethyl acetate, n-butanol successively, and then each extract was concentrated by a rotary evaporator and preserved at $4^{\circ} \mathrm{C}$.

2.2. Cell Line and Reagents. A549 cell line (human lung cancer cell line), Hela cell line (human cervical carcinoma cell line), SK-OV-3 cell line (human ovarian cancer), NIC-H1975 cell (human lung cancer cell line), K562 cell line (human leukemic cell line), S180 cell line (mouse sarcoma cell line), and H22 (mouse hepatoma cell line) were obtained from the ATCC (Manassas, VA, USA). SB203580 or SP600125 was purchased from Dalian Meilun Biology Co., Ltd. (Dalian, China). RPMI1640 medium was purchased from Gibco BRL, Life Technologies (USA). Fetal bovine serum (FBS) was purchased from Hyclone Laboratories (Logan, Utah, USA). The antibodies were purchased from Cell Signaling Technology. FITC-conjugated, Annexin V, and PI were purchased from BD Bioscience.

2.3. Experimental Animals. Immunodeficient $\mathrm{BALB} / \mathrm{c}$ male mice (BALB/c-nu/nu) aged 6-8 weeks were purchased from Shanghai Laboratory Animal Center of the Chinese Academy of Sciences. All experiments were approved by Ethical Committee of No. 1 Hospital Affiliated Yunnan University of Traditional Chinese Medicine.
2.4. Cell Viability Assay. MTT assay was used to evaluate antitumor activity, and detailed method was performed as previously described $[11,12]$. Label-free real-time detection technology was used for continuously monitoring tumor cells proliferation and viability. In brief, A549 and NICH1975 cells were seeded 96-well microtiter E-plate (Roche Applied Sciences), and the cells were treated with different concentrations of EAAC for $48 \mathrm{~h}$. The cell response to EAAC treatment was continuously monitored by xCELLigence SP system.

2.5. Antitumor Activity of EAAC In Vivo. BALB/c-nu/nu were injected s.c. into the right forelimb armpit with $4 \times 10^{6}$ NIC-H1975 lung tumor cells, and the mice were then randomly divided into three groups: vehicle control group, EAAC group, and Cyclophosphamide (CTX ) group. The Cyclophosphamide group was injected i.p. with $10 \mathrm{mg} / \mathrm{kg}$ Cyclophosphamide, EAAC group was orally administered $100 \mathrm{mg} / \mathrm{kg}$ EAAC, and vehicle control group was perfused with the same volume of $0.9 \%$ physiological saline. 21 days after the last drug administration, the mice were sacrificed, then tumor volume and weight were calculated.

2.6. Assessment of Apoptosis. Apoptosis was measured by Annexin V/PI-staining and Hoechst 33342 staining according to the method described previously [13] For Annexin V/ PI-staining assay, NIC-H1975 cells were harvested after EAAC or MAPK inhibitor treatment, and the cells were stained with FITC-conjugated Annexin V and PI for 15 min and then analyzed with a flow cytometer (FACSCanto ${ }^{\mathrm{TM}} \mathrm{II}$; BD Biosciences). For Hoechst dye staining, NIC-H1975 cells were stained with Hoechst33342 for 10 min after 24h EAAC treatment and were then photographed by fluorescent microscope.

2.7. Western Blotting Assay. Western blot was performed according to the procedures previously described [14]. Briefly, NIC-H1975 cells were treated with different concentrations of EAAC, and whole cell lysates were prepared. Proteins were electrophoresed through 10\% SDS-PAGE gel and transferred to the nitrocellulose membrane. The blots were blocked with 5\% BSA-TBST buffer and then incubated overnight at $4^{\circ} \mathrm{C}$ with primary antibodies. Membranes were washed and incubated with HRP-conjugated secondary antibody, and immune complexes were detected by enhanced chemiluminescence.

2.8. Statistical Analysis. Data were expressed as Mean \pm SD of experiments. Student's t-test was used to determine significance between two groups. $P<0.05$ was considered to be statistically significant.

\section{Result}

3.1. Ethyl Acetate Extract of Asclepias curassavica Exerted Best Antitumor Activity In Vitro. Cytotoxic activity of different extract of Asclepias curassavica was evaluated against human lung cancer cell line A549 and NIC-H1975, human cervical 
TABLE 1: $\mathrm{IC}_{50}$ values of different extract of Asclepias curassavica in different types of tumor cell lines after $48 \mathrm{~h}$ of treatment.

\begin{tabular}{lccccc}
\hline Component & & \multicolumn{2}{c}{ Cell lines $\left(\mathrm{IC}_{50}, \mu \mathrm{g} / \mathrm{mL}\right)^{a, b}$} & K562 & NIC-H1975 \\
\hline Water extract & A549 & Hela & SK-OV-3 & 21.97 & 113.00 \\
Chloroform extract & 14.72 & 1.96 & 5.92 & 3.45 & 1.75 \\
Ethyl acetate extract & 1.85 & 4.91 & 1.92 & 0.76 & 0.40 \\
Cisplatin & 0.66 & 0.43 & 12.44 & 8.95 & 5.43 \\
\hline
\end{tabular}

${ }^{a}$ Cytotoxicity as $\mathrm{IC}_{50}$ values for each cell line, the concentration of compound that inhibits $50 \%$ of the cell growth measured by MTT assay.

${ }^{b}$ Each value was reproduced in triplicate.

carcinoma Hela, human ovarian cancer SK-OV-3, and human leukemic cell line K562 by MTT assay, using cisplatin as the reference drug. The biological results of different extract of Asclepias curassavica were summarized in Table 1.

As shown in Table 1, different extracts of Asclepias curassavica display significant antitumor activities against a panel of human tumor cell lines, among which ethyl acetate extract of Asclepias curassavica possessed the highest cytotoxic activity in vitro (Table 1 ). The half maximal inhibitory concentration $\left(\mathrm{IC}_{50}\right)$ of A549, Hela, SK-OV-3, K562, and NIC-H1975 cells was calculated to be about $0.66,0.43,1.92$, 0.76 , and $0.40 \mu \mathrm{g} / \mathrm{ml}$, respectively. Thus, ethyl acetate extract of Asclepias curassavica was chosen as representative for further investigation of the antitumor activity and exploration of the underlying mechanism.

3.2. EAAC Caused Cytotoxicity in Dose-Dependent and TimeDependent Manner. To further explore therapeutic potential of EAAC, the antitumor activity of EAAC was investigated by a real-time cell analysis system (xCELLigence) monitoring A549, and NIC-H1975 cell lines. Unlike the conventional endpoint cell viability assays, the real-time cell detection system continuously monitors live cells in response to the exposure of EAAC and provides kinetic cell response information from the same cell population. The results showed that dose- and time-dependent cell responses to EAAC were clearly shown in A549 and NIC-H1975 cell lines (Figures 1(a)-1(c)). Consistent with the real-time cell detection system, EAAC caused cytotoxicity against A549 and NIC-H1975 in a dose-dependent manner. Based on the $\mathrm{IC}_{50}$, NIC-H1975 cells were treated with $0.5 \mu \mathrm{g} / \mathrm{ml}$ EAAC for $0,24,48$, and $72 \mathrm{~h}$, and the results exhibited that EAAC exerted strong cytotoxicity against NIC-H1975 in a time-dependent manner (Figure 1(d)).

\subsection{EAAC Exerts Significant Antitumor Effect on NIC-H1975} Tumors in BALB/c-nu/nu Mouse. EAAC showed significant antitumor activity in vitro as described above. In order to further evaluate in vivo antitumor effect of EAAC, BALB/cnu/nu mouse model of NIC-H1975 cell was established for measuring in vivo antitumoral effect. Twenty four hours after injected tumor, EAAC or vehicle was administered, i.e., Cyclophosphamide was injected i.p., the experiment was terminated after 3 weeks, and tumor volume and weight were calculated. The result indicated that, compared with vehicle group, tumor volume and weight were lower in EAAC treated group, and tumor inhibition rates on NIC-H1975 tumors in BALB/c-nu/nu mouse were $46.82 \%$ (Figure 2), implying administration of EAAC was an effective antitumor therapy in vivo.

3.4. EAAC Induced Apoptosis in NIC-H1975 Cell Lines. In order to detect the effect of EAAC on apoptosis, we then choose the EAAC-sensitive cell line NIC-H1975 for further study. EAAC induced apoptosis was detected using Hoechst33342 staining and Annexin V/PI-staining by flow cytometry. Flow cytometry analysis showed that EAAC significantly promoted the NIC-H1975 cell into early stage of apoptosis in a dose-dependent manner, and the proportion of apoptotic cells in control group was $2.86 \%$, while EAAC treatment significantly increased the proportion of apoptotic cells to be $16.50 \%$ (Figure 3(a)). Consistently, Hoechst33342 staining observed extensively chromatin condensation and nuclear fragmentation in NIC-H1975 cells after $1 \mu \mathrm{g} / \mathrm{ml}$ EAAC treatment, suggesting these cells underwent apoptosis (Figure 3(b)). These results indicated that EAAC can effectively induce apoptosis in NIC-H1975 cells.

\subsection{EAAC Treatment Regulates the Expression of Proteins} Involved in Apoptosis. To investigate the underlying mechanism of EAAC-induced apoptosis in NIC-H1975, we tested the activation of caspase $3 / 8 / 9$ and consequent PARP cleavage by western blot. Data showed EAAC exerted dose-depended effect on activation of these caspases leading to increased PARP cleavage (Figure 4(a)). In addition, Fas level increased following $2 \mu \mathrm{g} / \mathrm{ml}$ EAAC treatment (Figure $4(\mathrm{a})$ ). Together, these data indicated that both mitochondrial and death receptor pathways were involved in EAAC-induced apoptosis.

Antiapoptotic proteins are important candidates of regulating apoptosis. Bcl-2, XIAP, survivin, and Mcl-1 were checked in the induction of apoptosis in NIC-H1975 by EAAC. The result showed that EAAC significantly downregulated antiapoptotic proteins Bcl-2, XIAP, survivin, and Mcl-1. Additionally, proapoptosis proteins bak and bax were obviously upregulated after exposure to EAAC (Figure 4(b)).

3.6. EAAC Induced Apoptosis via Activating p38 and JNK $M A P K$ Signaling Pathways. In order to analyze the role of p38, ERK, and JNK MAPK signaling pathways in EAAC induced cancer cells apoptosis in NIC-H1975, the phosphorylation of p38, ERK, and JNK MAPK was determined 


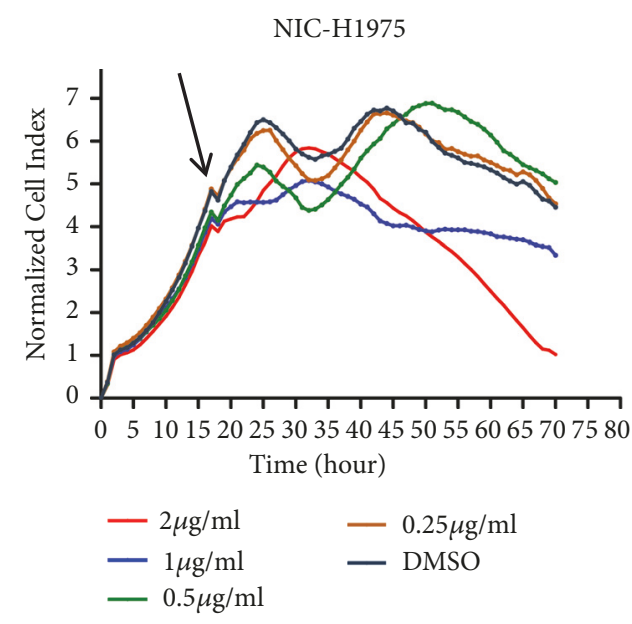

(a)

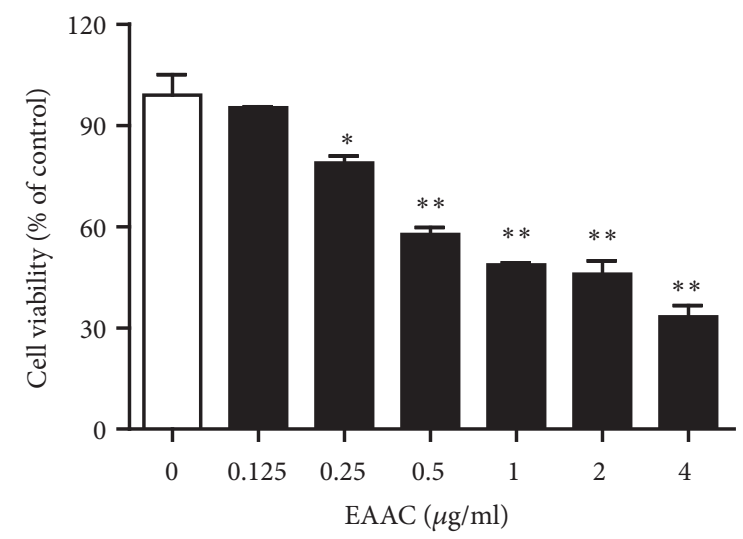

(c)

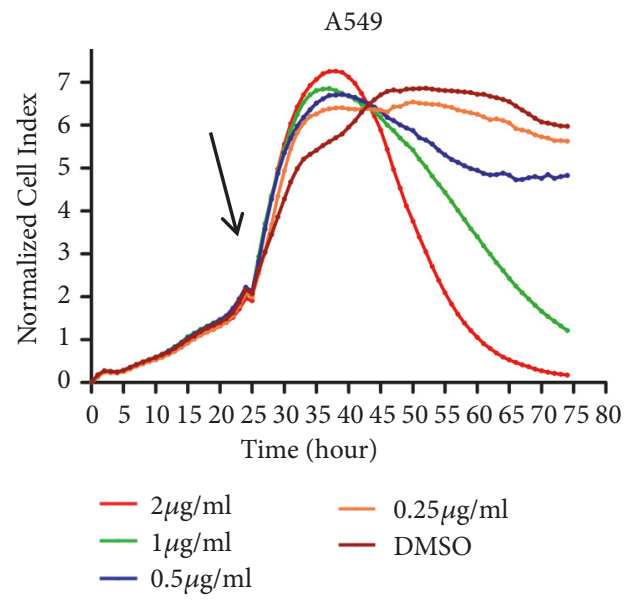

(b)

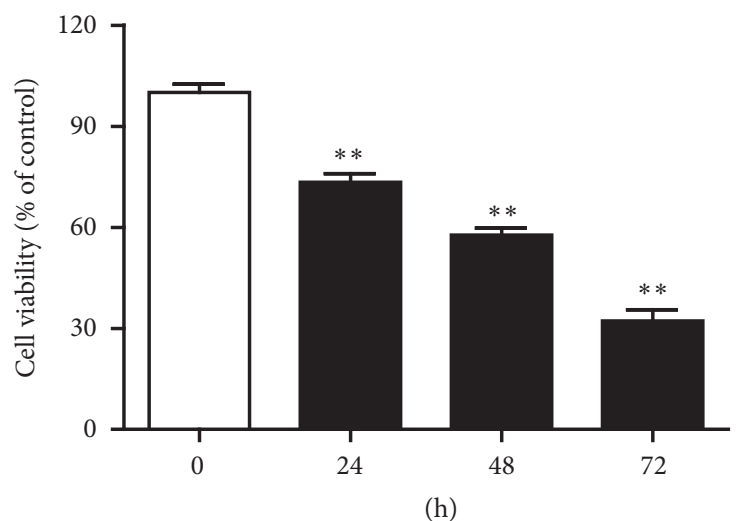

(d)

FIGURE 1: EAAC caused cytotoxicity in a dose-dependent manner and in a time-dependent manner. A549 and NIC-H1975 cells were seeded into 96-well microtiter E-plate. Then, these cells were treated with different concentrations of EAAC. The cell response to EAAC treatment was continuously monitored by xCELLigence SP system for 48h (Figures 1(a) and 1(b)). NIC-H1975 cells were seeded into 96-well microtiter plates, the cells were treated with various concentrations of EAAC for $48 \mathrm{~h}$, and cell viability was determined using a MTT assay (Figure 1(c)). NIC-H1975 cells were treated with $(0.5 \mu \mathrm{g} / \mathrm{ml})$ EAAC for the indicated times and cell viability was analyzed using a MTT assay (Figure $1(\mathrm{~d}))$. Results presented mean \pm s.e.m., $\mathrm{n}=3 . * P<0.05, * * P<0.01$ versus control group.

by western blot assay. NIC-H1975 cells were treated with EAAC for $24 \mathrm{~h}$, and then the cell lysates were immunoblotted with pan-phosphor-specific antibodies, western blot assay result showed that the phosphorylation of p38 and JNK was markedly increased after EAAC treatment, and there were no notable changes in ERK (Figure 5).

3.7. Inhibitor of $p 38$ and JNK Abrogated EAAC Induced Apoptosis. EAAC treatment significantly induced the phosphorylation of p38 and JNK as described above. In order to further ensure if p38 and JNK MAPK signaling pathways were involved in EAAC induced apoptosis, cells were pretreated with SB203580 or SP600125 prior to the addition of EAAC. SB203580, an inhibitor of p38, reduced the apoptosis induced by EAAC (Figure 6(a)). The SP600125 was a specific inhibitor for JNK signaling pathway. As shown in Figure 6(b), SP600125 treatment abrogated EAAC induced apoptosis.
These results are consistent with western blot results, implying that EAAC induced apoptosis through initializing the activation of p38 and JNK MAPK signaling pathway.

\section{Discussion}

Apoptosis or programmed cell death has been defined as a discrete sequence of morphological changes resulting in cell death with extensive dsDNA cleavage accompanied by chromatin compaction and segregation along the nuclear membrane [15]. Apoptosis is a central regulator of normal tissue homeostasis; it is essential for the elimination of redundant, damaged, and infected cells. In particular, apoptosis represented a fundamental antineoplastic mechanism preventing tumorigenesis of normal cells. Therefore, induction of tumor cell apoptosis was considered as an effective way of cancer therapy. 


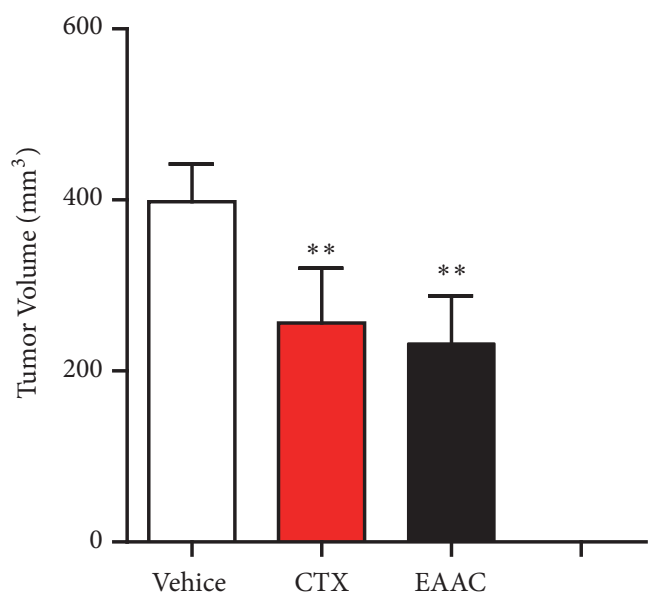

(a)

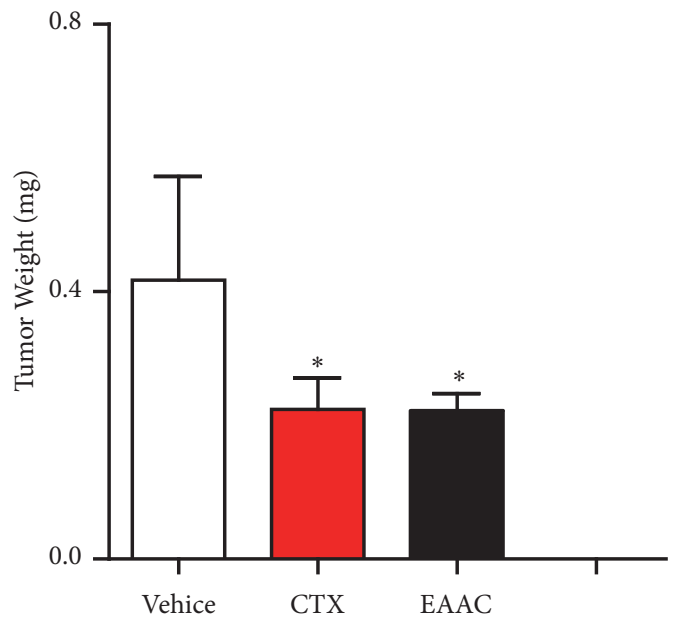

(b)

Figure 2: EAAC exerts significant antitumoral effect on NIC-H1975 tumors in BALB/c-nu/nu mouse. Nude mice (BALB/c nu/nu) were injected subcutaneously into the right forelimb armpit with $4 \times 10^{6}$ NIC-H1975 lung tumor cells, $24 \mathrm{~h}$ after injected tumor, and EAAC or vehicle was administered via intragastric administration. The experiment was terminated after 3 weeks, the mice were sacrificed, then tumor volume (Figure 2(a)) and tumor weight (Figure 2(b)) were calculated. Results presented mean \pm s.e.m., $\mathrm{n}=5 . * P<0.05, * * P<0.01$ versus vehicle group.
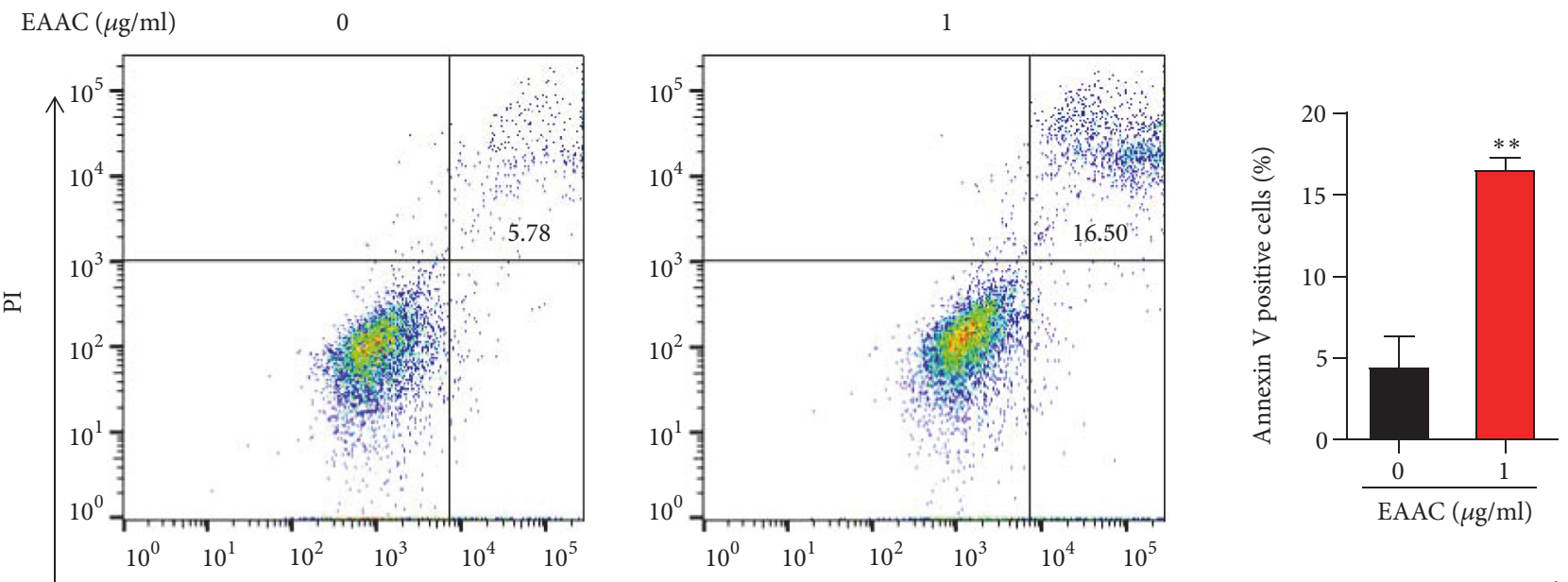

Annexin V

(a)

$\operatorname{EAAC}(\mu \mathrm{g} / \mathrm{ml})$

0

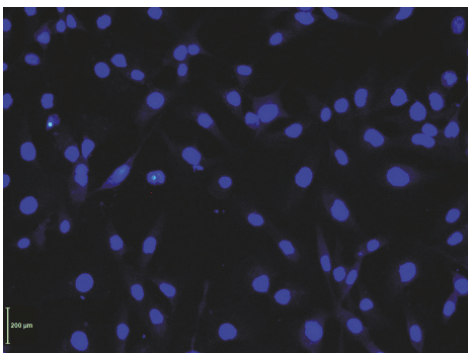

0.5

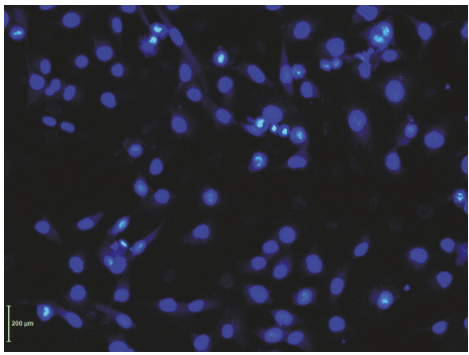

(b)

FIGURE 3: EAAC induced apoptosis in NIC-H1975 cells. NIC-H1975 cells were treated with various concentrations of EAAC for 24h. (a) The cells were harvested and apoptosis was measured by an Annexin V-FITC/PI. (b) The cells were stained with Hoechst 33342 for 10 min after $24 \mathrm{~h}$ EAAC treatment and were then photographed by fluorescent microscope. The result presented representative of three individual experiments. 


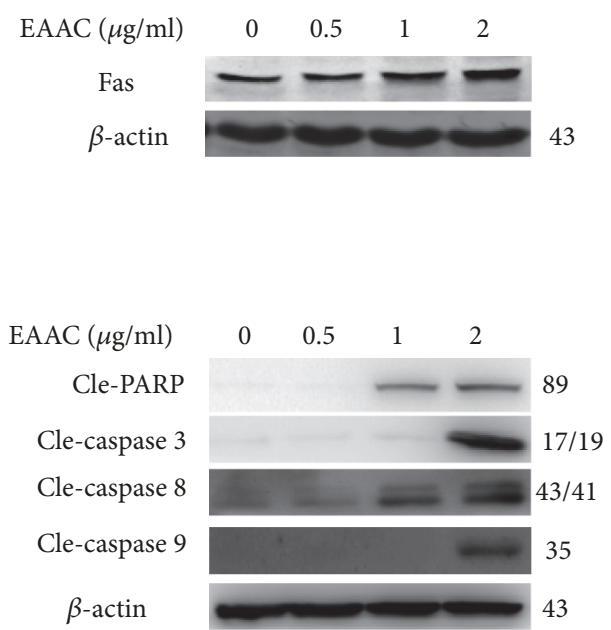

(a)

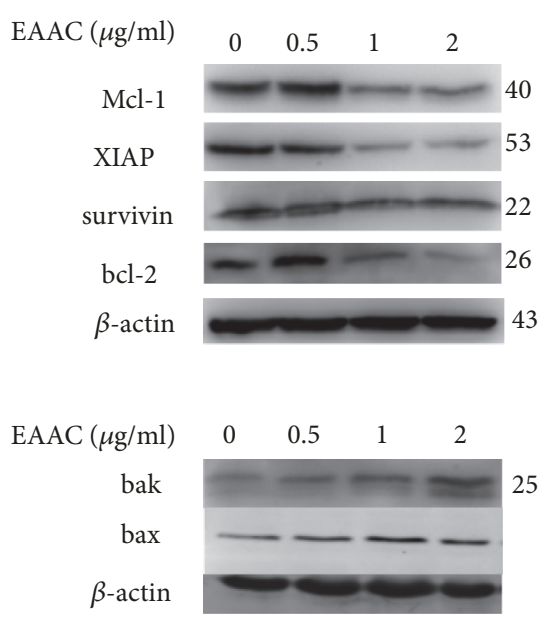

(b)

FIGURE 4: EAAC treatment regulates the expression of proteins involved in apoptosis. NIC-H1975 cells were exposed to different concentrations of EAAC for $48 \mathrm{~h}$, and whole cell extracts were obtained and determined by western blot assay using indicated antibody for assessing apoptosisrelated protein. The result presented representative of three individual experiments.

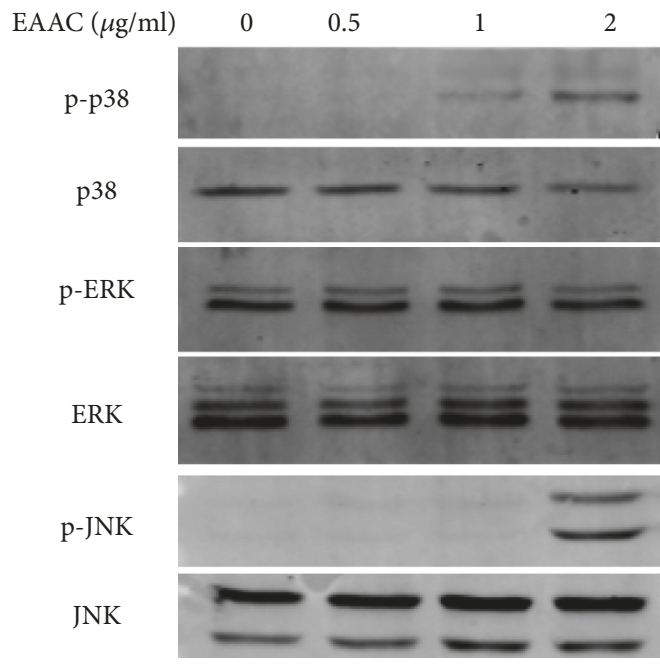

FIGURE 5: EAAC induced apoptosis via the MAPK pathway. NICH1975 cells were treated with different concentrations of EAAC, and the cell lysates were immunoblotted with pan-/phosphor-specific antibodies. The result presented representative of three individual experiments.

Asclepias curassavica L. (Asclepiadaceae) is a traditional medicinal plant, which was used as treatment for tumor, hemostatic disorders, inflammation, and pain in traditional Chinese medical practice; meanwhile, curassavica powder is also used to treat abdominal tumors in traditional Indian medicine [16-19]. Although the research about antitumor effect of Asclepias curassavica L. has made some progress over recent decade, their underlying molecular mechanism remains largely unknown.

In the present study, ethyl acetate extract of Asclepias curassavica exerted potent antitumoral effect both in vitro and in vivo. EAAC induced apoptosis was detected in NIC-H1975 cell by using flow cytometry, and the results indicated that EAAC treatment significantly promoted the NIC-H1975 cell into early stage of apoptosis with a dose-dependent manner, suggesting that EAAC exerted cytotoxicity in NIC-H1975 cell caused by inducing apoptosis.

Many chemotherapeutic agents have been thought as modulators in apoptosis-inducing signal transduction pathways, which contain the mitochondrial pathway, external death receptor pathway, and endoplasmic reticulum pathway [20]. Among them, Fas and FasL are mainly involved in the external death receptor pathway [21, 22]. Depending on the binding of Fas-associated protein to the death domain, caspase family cascade reactions are induced, which give rise to the degradation of DNA fragments and apoptosis [23]. It was considered that the expression level of Fas protein may be implied in the malignancy and development stage of tumors. Here, Fas protein expression was increased after $2 \mu \mathrm{g} / \mathrm{ml}$ EAAC treatment. Furthermore, EAAC treatment also activated caspase 3 . These results indicated that EAAC induced NIC-H1975 cell apoptosis might be through upregulating the expression of caspase 3 and Fas.

It has been noted that apoptosis was regulated by the activation of the caspase family; it occurs via the intrinsic or mitochondrial pathway and the extrinsic or death receptor pathway $[24,25]$. The intrinsic pathway results in cytochrome c releasing from the mitochondrial into cytosol and thus activating the initiator caspase 9. The extrinsic apoptotic pathway results from the activation of death-domain receptors and activates the initiator caspase 8 . Both extrinsic and intrinsic apoptotic pathways activate executioner caspase 3 via cleavage and eventually lead to apoptosis [26,27]. Our data showed EAAC treatment significantly induced activation of caspase 8 , caspase 9 , and caspase 3 at 24 h in the NIC-H1975 cell, by which increase PARP cleavage. These results suggested that EAAC induced apoptosis through caspase-dependent pathway in the NIC-H1975 cell. 
(a)
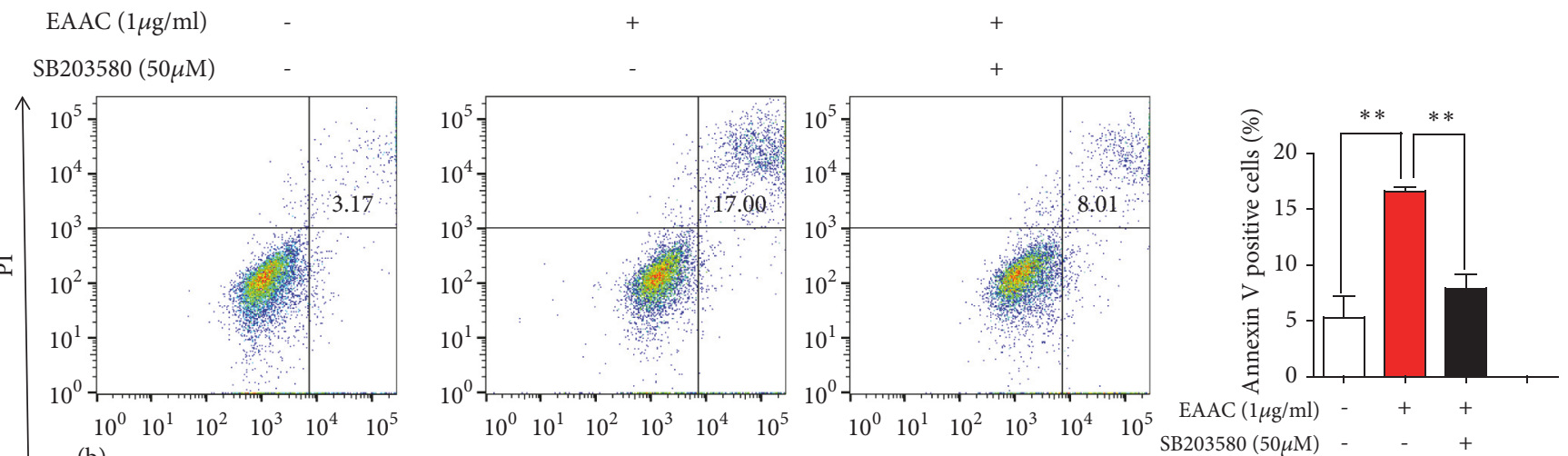

(b)
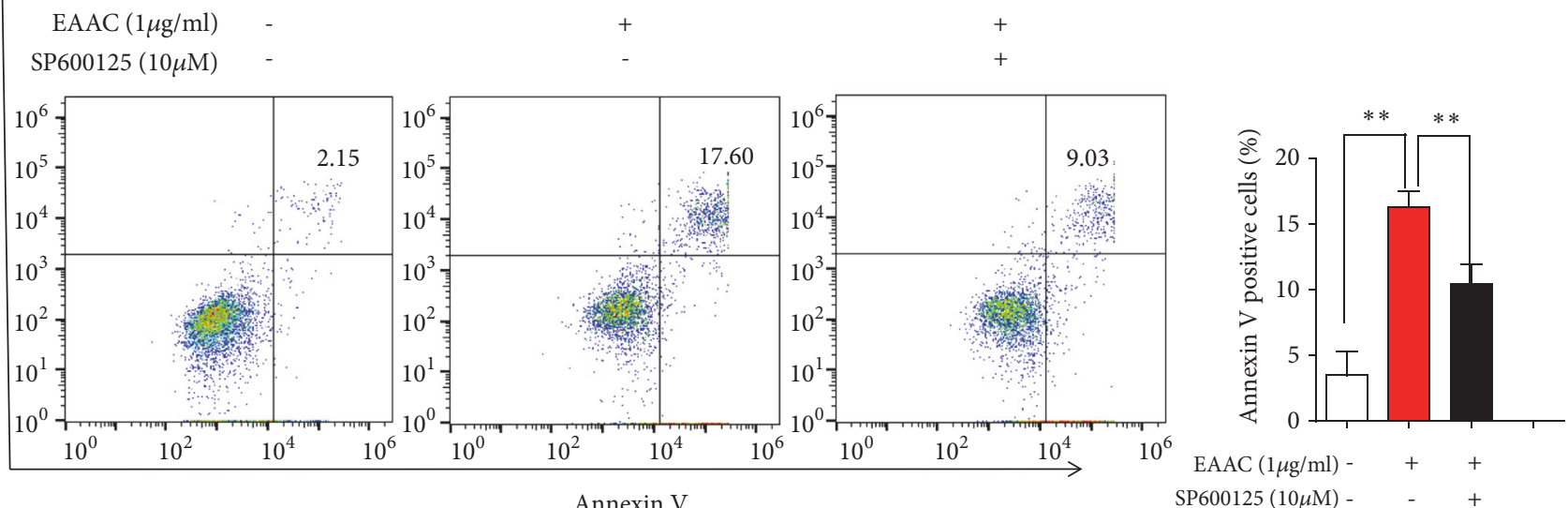

FIGURE 6: Inhibitor of p38 and JNK abrogated EAAC induced apoptosis. NIC-H1975 cells were pretreated with SB203580 or SP600125 prior to the addition of EAAC, then these cells were treated with various concentrations of EAAC for $24 \mathrm{~h}$. The cells were harvested and apoptosis was measured by an Annexin V-FITC/PI. Results presented mean \pm s.e.m., $\mathrm{n}=3 . * P<0.05, * * P<0.01$ versus EAAC treated group.

Many death and survival genes were involved in apoptosis, and members of the Bcl-2 family played a key role in the mitochondrial-mediated cell death pathway [28]. The proapoptotic bcl-2 family members had more increase than antiapoptotic bcl-2 family members, and the formation of pores in the outer mitochondrial membrane results in the releasing of apoptogenic mitochondrial proteins, which in turn activates caspases to induce apoptosis [29]. Our study revealed EAAC significantly downregulated antiapoptotic protein Bcl-2, XIAP, survivin, and Mcl-1. More importantly, EAAC treatment upregulated the proapoptotic protein bak.. The finding indicated that EAAC acts in both intrinsic and extrinsic apoptotic pathways via the regulation of antiapoptotic proteins and proapoptotic proteins. MAPK superfamily has implication in cell proliferation, differentiation, invasion, and apoptosis [7, 30,31]. The MAPK signaling pathway could be activated by diverse extracellular stimuli and has a central role in cell apoptosis [32-34]. Extensive studies have shown that activation of ERK by compounds generates antiproliferative effects such as apoptosis, senescence in cancer cells, by which ERK activate apoptotic enzymes or phosphorylate transcription factors to regulate the expression of proapoptotic genes $[35,36]$. JNK is a key regulator of apoptosis [10]. JNK activation contributes to TNF- $\alpha$ and
UV-induced apoptosis, but it can suppress apoptosis phosphorylation of the proapoptotic Bcl-2 family protein BAD. Thus, in the regulation of apoptosis, JNK serves as doubleedged sword, depending on cell type, nature of the death stimulus, duration of its activation, and the activity of other signaling pathways [37]. In this study, phosphorylation of p38 and JNK was remarkably induced by EAAC; however, there were no notable changes in ERK1/2. Moreover, we observed that inhibition of p38 and JNK MAPK by pharmacological inhibitors (SB203580 and SP600125) abrogated EAAC-induced apoptosis, suggesting EAAC induced apoptosis through regulation of p38 MAPK and JNK signaling pathways.

In this study, we establish that EAAC had potent antitumor effect both in vitro and in vivo by triggering cell apoptotic pathway. These data paved the way for further evaluation of EAAC as a new anticancer herb in Chinese traditional medical practice.

\section{Data Availability}

The data used to support the findings of this study are available from the corresponding author upon request. 


\section{Conflicts of Interest}

The authors disclose no conflicts of interest regarding this study.

\section{Authors' Contributions}

$\mathrm{Xi}$ Zheng and Ying Xu contributed equally to this work.

\section{Acknowledgments}

This work was funded by the Natural Science Foundation of China (Grant no. 81460624), Yunnan Provincial Science and Technology Department-Applied Basic Research Joint Special Fund of Yunnan University of Traditional Chinese Medicine (Grant nos. 2018FF001(-077) and 2017FF117(041)), The Applied Basic Research Program of Yunnan Province, China (Grant no. 2015FB199), and Yunnan Applicative and Basic Research Program (Grant no. 2018FY001-001).

\section{References}

[1] Jiangsu New Medical Colledge, The Dictionary of Traditional Chinese Materia Medica, Shanghai Science and Technology Press, Shanghai, China, 1997.

[2] D. Hanahan and R. A. Weinberg, "Hallmarks of cancer: the next generation," Cell, vol. 144, no. 5, pp. 646-674, 2011.

[3] M. Certo, V. D. G. Moore, M. Nishino et al., "Mitochondria primed by death signals determine cellular addiction to antiapoptotic BCL-2 family members," Cancer Cell, vol. 9, no. 5, pp. 351-365, 2006.

[4] R. J. Youle and A. Strasser, "The BCL-2 protein family: opposing activities that mediate cell death," Nature Reviews Molecular Cell Biology, vol. 9, no. 1, pp. 47-59, 2008.

[5] A. Ruefli-Brasse and J. C. Reed, "Therapeutics targeting Bcl-2 in hematological malignancies," Biochemical Journal, vol. 474, no. 21, pp. 3643-3657, 2017.

[6] M. Guha, S. Kumar, V. Choubey, P. Maity, and U. Bandyopadhyay, "Apoptosis in liver during malaria: role of oxidative stress and implication of mitochondrial pathway," The FASEB Journal, vol. 20, no. 8, pp. 1224-1226, 2006.

[7] G. L. Johnson and R. Lapadat, "Mitogen-activated protein kinase pathways mediated by ERK, JNK, and p38 protein kinases," Science, vol. 298, no. 5600, pp. 1911-1912, 2002.

[8] C. Bradham and D. R. McClay, "p38 MAPK in development and cancer," Cell Cycle, vol. 5, no. 8, pp. 824-828, 2006.

[9] L. Hui, L. Bakiri, E. Stepniak, and E. F. Wagner, "p38 $\alpha$ : a suppressor of cell proliferation and tumorigenesis," Cell Cycle, vol. 6, no. 20, pp. 2429-2433, 2007.

[10] Z. Xia, M. Dickens, J. Raingeaud, R. J. Davis, and M. E. Greenberg, "Opposing effects of ERK and JNK-p38 MAP kinases on apoptosis," Science, vol. 270, no. 5240, pp. 1326-1331, 1995.

[11] Y. Ma, X. Zheng, H. Gao, C. Wan, G. Rao, and Z. Mao, "Design, synthesis, and biological evaluation of novel benzofuran derivatives bearing $\mathrm{N}$-aryl piperazine moiety," Molecules, vol. 21, no. 12, 2016.

[12] S. Kustermann, F. Boess, A. Buness et al., "A label-free, impedance-based real time assay to identify drug-induced toxicities and differentiate cytostatic from cytotoxic effects," Toxicology in Vitro, vol. 27, no. 5, pp. 1589-1595, 2013.
[13] P. Liu, S. Xu, M. Zhang et al., "Anticancer activity in human multiple myeloma U266 cells: Synergy between cryptotanshinone and arsenic trioxide," Metallomics, vol. 5, no. 7, pp. 871-878, 2013.

[14] Y. Ge, R. Cheng, Y. Zhou et al., "Cryptotanshinone induces cell cycle arrest and apoptosis of multidrug resistant human chronic myeloid leukemia cells by inhibiting the activity of eukaryotic initiation factor 4E," Molecular and Cellular Biochemistry, vol. 368, no. 1-2, pp. 17-25, 2012.

[15] P. A. Furth, "Introduction: mammary gland involution and apoptosis of mammary epithelial cells.," Journal of Mammary Gland Biology and Neoplasia, vol. 4, no. 2, pp. 123-127, 1999.

[16] A. Al-Snafi, "The Chemical constituents and pharmacological effects of Asclepias carum carvi-A review," Asian Journal of Pharmaceutical and Clinical Research, vol. 5, pp. 72-82, 2015.

[17] R. Sundararajan and R. Koduru, "Asclepias curassavica: A review of ethnomedical, phytochemical and pharmacological information," Indo American Journal of Pharmaceutical Research, vol. 4, no. 4, pp. 2151-2169, 2014.

[18] C. Liggieri, W. Obregón, S. Trejo, and N. Priolo, "Biochemical analysis of a papain-like protease isolated from the latex of Asclepias curassavica L.," Acta Biochimica et Biophysica Sinica, vol. 41, no. 2, pp. 154-162, 2009.

[19] C. Liggieri, M. C. Arribére, S. A. Trejo, F. Canals, F. X. Avilés, and N. S. Priolo, "Purification and biochemical characterization of asclepain c I from the latex of Asclepias curassavica L.," The Protein Journal, vol. 23, no. 6, pp. 403-411, 2004.

[20] P. Aryal, K. Kim, P.-H. Park, S. Ham, J. Cho, and K. Song, "Baicalein induces autophagic cell death through AMPK/ULK1 activation and downregulation of mTORC1 complex components in human cancer cells," FEBS Journal, vol. 281, no. 20, pp. 4644-4658, 2014.

[21] H. Du, Y. Huang, X. Hou et al., "Two novel camptothecin derivatives inhibit colorectal cancer proliferation via induction of cell cycle arrest and apoptosis in vitro and in vivo," European Journal of Pharmaceutical Sciences, vol. 123, pp. 546-559, 2018.

[22] D. Wu, D. Zhao, Y. Li et al., "Antitumor potential of a novel camptothecin derivative, ZBH-ZM-06," Oncology Reports, vol. 39, no. 2, pp. 871-879, 2018.

[23] W. Tang, Q. Liu, X. Wang et al., "Involvement of caspase 8 in apoptosis induced by ultrasound-activated hematoporphyrin in sarcoma 180 cells in vitro," Journal of Ultrasound in Medicine, vol. 27, no. 4, pp. 645-656, 2008.

[24] F. G. Blankenberg, "Apoptosis imaging: Anti-cancer agents in medicinal chemistry," Anti-Cancer Agents in Medicinal Chemistry, vol. 9, no. 9, pp. 944-951, 2009.

[25] N. Pabla and Z. Dong, "Cisplatin nephrotoxicity: mechanisms and renoprotective strategies," Kidney International, vol. 73, no. 9, pp. 994-1007, 2008.

[26] A. Philchenkov, "Caspases: Potential targets for regulating cell death," Journal of Cellular and Molecular Medicine, vol. 8, no. 4, pp. 432-444, 2004.

[27] J. M. Zapata, K. Pawlowski, E. Haas, C. F. Ware, A. Godzik, and J. C. Reed, "A diverse family of proteins containing tumor necrosis factor receptor-associated factor domains," The Journal of Biological Chemistry, vol. 276, no. 26, pp. 24242-24252, 2001.

[28] D. Hockenbery, G. Nunez, C. Milliman, R. D. Schreiber, and S. J. Korsmeyer, " $\mathrm{Bcl}-2$ is an inner mitochondrial membrane protein that blocks programmed cell death," Nature, vol. 348, no. 6299, pp. 334-336, 1990.

[29] J. M. Adams and S. Cory, "The Bcl-2 protein family: arbiters of cell survival," Science, vol. 281, no. 5381, pp. 1322-1326, 1998. 
[30] J. Matsukawa, A. Matsuzawa, K. Takeda, and H. Ichijo, "The ASK1-MAP kinase cascades in mammalian stress response," The Journal of Biochemistry, vol. 136, no. 3, pp. 261-265, 2004.

[31] A. S. Dhillon, S. Hagan, O. Rath, and W. Kolch, "MAP kinase signalling pathways in cancer," Oncogene, vol. 26, no. 22, pp. 3279-3290, 2007.

[32] S.-J. Park and I.-S. Kim, "The role of p38 MAPK activation in auranofin-induced apoptosis of human promyelocytic leukaemia HL-60 cells," British Journal of Pharmacology, vol. 146, no. 4, pp. 506-513, 2005.

[33] Y. Zhan, K. Gong, C. Chen, H. Wang, and W. Li, "P38 MAP kinase functions as a switch in MS-275-induced reactive oxygen species-dependent autophagy and apoptosis in Human colon Cancer cells," Free Radical Biology \& Medicine, vol. 53, no. 3, pp. 532-543, 2012.

[34] R. Pradhan, G. Singhvi, S. K. Dubey, G. Gupta, and K. Dua, "MAPK pathway: a potential target for the treatment of nonsmall-cell lung carcinoma," Future Medicinal Chemistry, 2019.

[35] S. Cagnol and J. C. Chambard, "ERK and cell death: Mechanisms of ERK-induced cell death-apoptosis, autophagy and senescence," FEBS Journal, vol. 277, no. 1, pp. 2-21, 2010.

[36] H. Randhawa, K. Kibble, H. Zeng, M. P. Moyer, and K. M. Reindl, "Activation of ERK signaling and induction of colon cancer cell death by piperlongumine," Toxicology in Vitro, vol. 27, no. 6, pp. 1626-1633, 2013.

[37] J. Liu and A. Lin, "Role of JNK activation in apoptosis: a doubleedged sword," Cell Research, vol. 15, no. 1, pp. 36-42, 2005. 


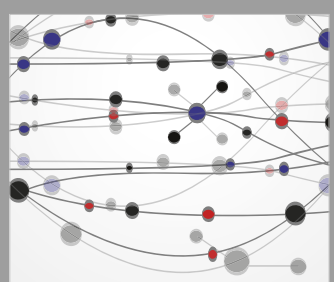

The Scientific World Journal
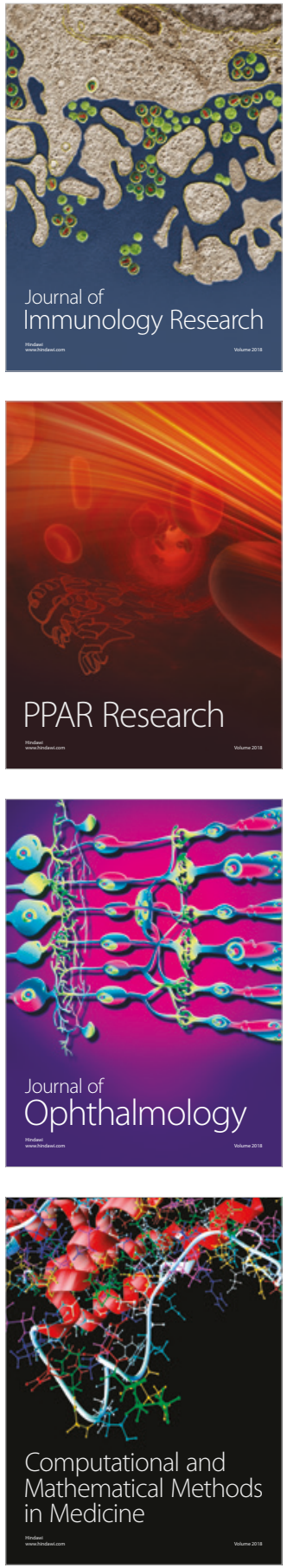

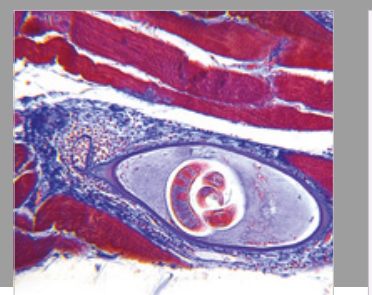

Gastroenterology Research and Practice

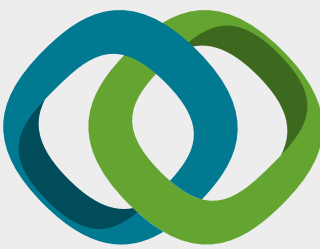

\section{Hindawi}

Submit your manuscripts at

www.hindawi.com
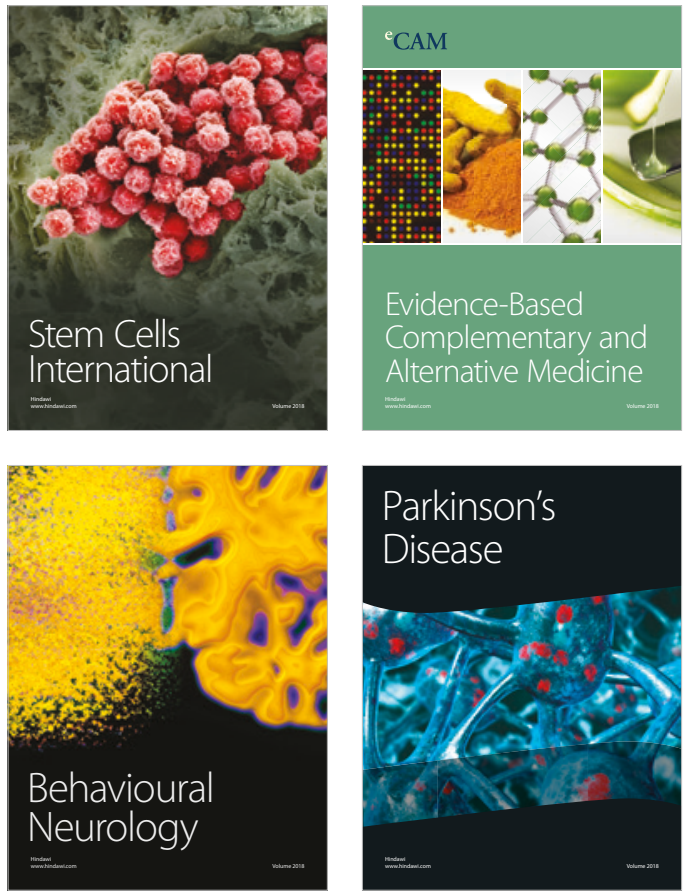

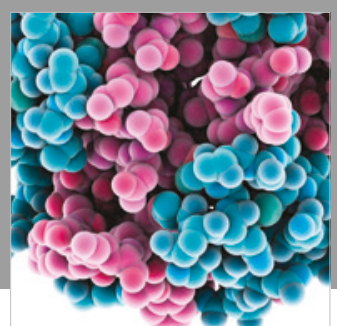

ournal of

Diabetes Research

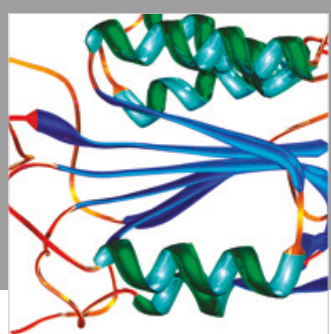

Disease Markers
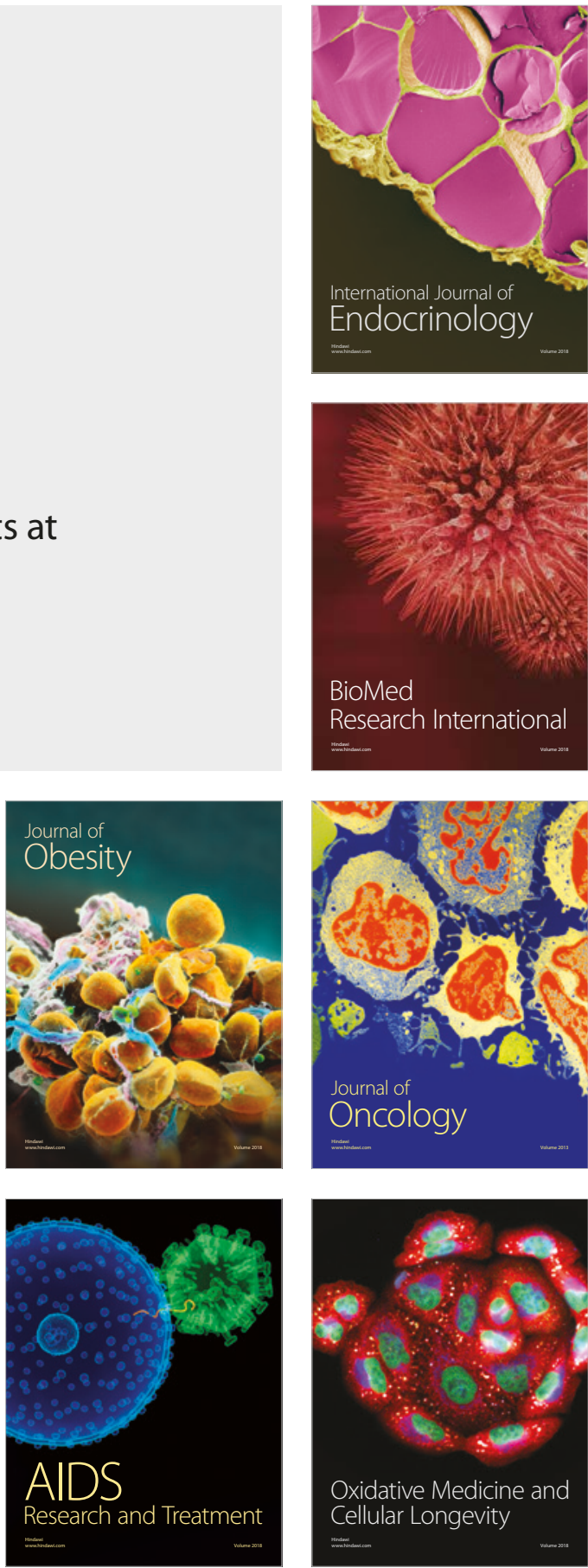